\title{
COMPRESSIBLE ANALYSIS OF BÉNARD CONVECTION OF MAGNETO ROTATORY COUPLE-STRESS FLUID
}

\author{
C.B. MEHTA* \\ Department of Mathematics \\ Govt. Excellence Degree College Sanjauli \\ Distt Shimla (H.P), INDIA \\ E-mail: chanderbmehta@gmail.com \\ M. SINGH \\ Department Mathematics \\ Govt. PG College Seema (Rohru) \\ Distt Shimla(H.P)-171207, INDIA \\ E-mail: mahinder_singh91@rediffmail.in
}

\begin{abstract}
Thermal Instability (Benard's Convection) in the presence of uniform rotation and uniform magnetic field (separately) is studied. Using the linearized stability theory and normal mode analyses the dispersion relation is obtained in each case. In the case of rotatory Benard's stationary convection compressibility and rotation postpone the onset of convection whereas the couple-stress have duel character onset of convection depending on rotation parameter. While in the absence of rotation couple-stress always postpones the onset of convection. On the other hand, magnetic field on thermal instability problem on couple-stress fluid for stationary convection couple-stress parameter and magnetic field postpones the onset of convection. The effect of compressibility also postpones the onset of convection in both cases as rotation and magnetic field. Graphs have been plotted by giving numerical values to the parameters to depict the stationary characteristics. Further, the magnetic field and rotation are found to introduce oscillatory modes which were non-existent in their absence and then the principle of exchange of stability is valid. The sufficient conditions for non-existence of overstability are also obtained.
\end{abstract}

Key words: couple-stress fluid, compressibility, rotation, magnetic field and thermal convection.

\section{Introduction}

Thermal instability of a fluid layer heated from below plays an important role in geophysics, oceanography, atmospheric physics etc., and has been investigated by many authors, e.g. Bénard [1], Rayleigh [2], Jeffreys [3]. A detailed account of the theoretical and experimental studies of so called Bénard convection in Newtonian fluids has been given by Chandrasekhar [4]. The Boussinesq approximation, which states that the density can be treated as a constant in all terms of the equations of motion except the external force term has been used throughout.

With the growing importance of non-Newtonian fluids in modern technology and industry, the investigations of such fluids are desirable. The presence of small amounts of additives in a lubricant can improve bearing performance by increasing the lubricant viscosity and thus producing an increase in the load capacity. These additives in a lubricant also reduce the coefficient of friction and increase the temperature range in which the bearing can operate. A number of theories of the microcontinuum have been postulated and applied (Lai et al. [5]; Walicka [6]).

\footnotetext{
${ }^{*}$ To whom correspondence should be addressed
} 
The theory of couple-stress fluid has been formulated by Stokes [7]. According to the theory of Stokes [7], couple-stresses are found to appear in noticeable magnitudes in fluids with very large molecules. One of the applications of couple-stress fluid is, therefore, its use to the study of the mechanisms of lubrication of synovial joints, which has become the object of scientific research. A human joint is a dynamically loaded bearing, which has articular cartilage as the bearing and synovial fluid as the lubricant. The shoulder, hip, knee and ankle joints are the loaded-bearing synovial joints of the human body and these joints have a low friction coefficient and negligible wear. Nason et al. [8] found that any instability, which is deleterious to certain biochemical separations, can be suppressed by rotation in the ultracentrifuge.

When the fluids are compressible, the equations governing the system become quite complicated. Spiegel and Veronis [9], have simplified the set of equations governing the flow of compressible fluids under the assumption that the depth of the fluid layer is much smaller than the scale height as defined by them, if only motions of infinitesimal amplitude are considered.

Walicki and Walicka [10] modeled synovial fluid as couple-stress fluid in human joints. The synovial fluid is the natural lubricant of joints of the vertebrates. The detailed description of the joint lubrication has very important practical lubrications. Practically all diseases of joints are caused by or connected with a malfunction of the lubrication. The efficiency of the physiological joint lubrication is caused by several mechanisms. The synovial fluid is, due to its content of the hyaluronic acid, a fluid of high viscosity, similar to a gel. Goel et al. [11] studied the hydromagnetic stability of an unbounded couple-stress binary fluid mixture under rotation with vertical temperature and concentration gradients. Kumar et al. [12] considered the thermal instability of a layer of couple-stress fluid acted on by uniform rotation and found that, for stationary convection, the rotation has a stabilizing effect, whereas the couplestress has both stabilizing and destabilizing effects. The use of magnetic field is being made for the clinical purposes in detection and cure of certain diseases with the help of magnetic field devices/ instruments. Singh and Kumar [14], have studied magneto and rotatory thermosolutal convection incompressible couple-stress fluid in porous medium, found that stable solute gradient, magnetic field and couple-stress parameter have stabilizing effects, where in rotation permeability and couple-stress parameter have both stabilizing as well as destabilizing effects on the system. In one another study Singh et al. [15] found that stable solute gradient, magnetic filed, couple-stress parameter have postpones the onset of convection where as rotation hastens the onset of convection. Also Singh and Kumar [16], in one another study found that stable solute gradient, magnetic filed and couple-stress parameter have stabilizing effect on the system.

Keeping in mind the importance of non-Newtonian fluids and convection in fluid heated from below, the present paper is devoted to study the compressible couple-stress fluid heated from below in presence of rotation and magnetic field.

\section{Effect of rotation}

\subsection{Formulation of the problems and perturbation equations}

Consider an infinite horizontal layer of a couple-stress fluid of depth $\mathrm{d}$ which is acted on by uniform rotation $\Omega(0,0, \Omega)$ and a gravity force $\boldsymbol{g}=(0,0,-g)$. This layer is heated from below so that a steady $\beta(=|d T / d z|)$ is maintained. Let $\Gamma_{i j}, \tau_{i j}, e_{i j}, \delta_{i j}, \mu, \mu^{\prime}, v_{i}$ and $x_{i}$ respectively, denote the stress tensor, shear stress tensor, rate-of-strain tensor, Kronecker delta, viscosity, couple-stress viscosity, viscosity vector and position vector. The constitutive relations for the couple-stress fluids are 


$$
\begin{aligned}
& \Gamma_{i j}=-p \delta_{i j}+\tau_{i j}, \quad \tau_{i j}=2\left(\mu-\mu^{*} \nabla^{2}\right) e_{i j}, \\
& e_{i j}=\frac{1}{2}\left(\frac{\partial v_{i}}{\partial x_{j}}+\frac{\partial v_{j}}{\partial x_{i}}\right) .
\end{aligned}
$$

The conditions on a free surface are the vanishing of tangential stresses $\tau_{x z}$ and $\tau_{y z}$, which yield

$$
\begin{aligned}
& \tau_{\mathrm{xz}}=\left(\mu-\mu^{*} \nabla^{2}\right)\left(\frac{\partial u}{\partial z}+\frac{\partial w}{\partial x}\right)=0, \\
& \tau_{\mathrm{yz}}=\left(\mu-\mu^{*} \nabla^{2}\right)\left(\frac{\partial v}{\partial z}+\frac{\partial w}{\partial y}\right)=0 .
\end{aligned}
$$

Since $w$ vanishes for all $x$ and $y$ on the bounding surface, it follows from Eqs (2.2) and (2.3) that

$$
\left(\mu-\mu^{*} \nabla^{2}\right) \frac{\partial u}{\partial z}=0, \quad\left(\mu-\mu^{*} \nabla^{2}\right) \frac{\partial v}{\partial z}=0
$$

We shall suppose the stars $(*)$ in distances for convenience hereafter.

Let $\boldsymbol{v}(u, v, w), \theta, \gamma, \delta p$ and $\delta \rho$ denote respectively the perturbations in velocity $(0,0,0)$, temperature $T$, solute concentration $C$, pressure $p$ and density $\rho$. Since the non-linear theories attempt to allow for the finite amplitudes of the perturbations, we suppose that the various physical variables describing the flow suffer small (infinitesimal) increments and as a consequence, we neglect all product and powers (higher than the first) of the increments and retain only terms that are linear and the linear stabilizing theory, for mathematical simplicity, is applied. Then the linearized perturbation equations, relevant to the problem (Stokes, [7]; Sharma, [13]), are

$$
\begin{aligned}
& \frac{\partial \boldsymbol{v}}{\partial t}=-\frac{1}{\rho_{m}} \nabla \delta p+\boldsymbol{g} \frac{\partial \rho}{\rho_{m}}+\left(v-\frac{\mu^{*}}{\rho_{m}} \nabla^{2}\right) \nabla^{2} \boldsymbol{v}+2(\boldsymbol{v} \times \boldsymbol{\Omega}), \\
& \nabla . \boldsymbol{v}=0, \\
& \frac{\partial \theta}{\partial t}=\left(\beta-\frac{g}{c_{p}}\right) w+\kappa \nabla^{2} \theta .
\end{aligned}
$$

Here $v, c_{p}$ and $\kappa$ stand for the kinematic viscosity, specific heat at constant pressure and thermal diffusivity respectively. From the equation of continuity (2.6) differentiated with respect to $z$, we conclude that

$$
\left[\mu-\mu^{*}\left(\frac{\partial^{2}}{\partial x^{2}}+\frac{\partial^{2}}{\partial y^{2}}+\frac{\partial^{2}}{\partial z^{2}}\right)\right] \frac{\partial^{2} w}{\partial z^{2}}=0
$$

The equation of state is 


$$
\rho=\rho_{m}\left[1-\alpha\left(T-T_{0}\right)\right]
$$

where $\alpha, \rho_{m}$ and $T_{0}$ are respectively, the coefficient of thermal expansion, the reference density and reference temperature at the lower boundary, therefore the change in density $\delta \rho$ caused by the perturbation $\theta$ in temperature is given by

$$
\delta \rho=-\rho_{m} \alpha \theta .
$$

Within the framework of the Boussinesq approximation, Eqs (2.5)- (2.7) and (2.10) yield

$$
\begin{aligned}
& \frac{\partial}{\partial t} \nabla^{2} w-\left(v-\frac{\mu^{*}}{\rho_{m}} \nabla^{2}\right) \nabla^{4} w-g \alpha\left(\frac{\partial^{2}}{\partial x^{2}}+\frac{\partial^{2}}{\partial y^{2}}\right) \theta+2 \Omega \frac{\partial \zeta}{\partial z}=0, \\
& \frac{\partial \zeta}{\partial t}-\left(v-\frac{\mu^{*}}{\rho_{m}} \nabla^{2}\right) \nabla^{2} \zeta=2 \Omega \frac{\partial w}{\partial z}, \\
& \left(\frac{\partial}{\partial t}-\kappa \nabla^{2}\right) \theta=\left(\beta-\frac{g}{c_{p}}\right) w
\end{aligned}
$$

where $\quad \nabla^{2}=\frac{\partial^{2}}{\partial x^{2}}+\frac{\partial^{2}}{\partial y^{2}}+\frac{\partial^{2}}{\partial z^{2}}$. the form

We now analyze the disturbances into normal modes, assuming that the perturbation quantities are of

$$
[w, \theta, \zeta]=[W(z), \Theta(z), Z(z)] \exp \left(i k_{x} x+i k_{y} y+n t\right)
$$

where $k_{x}, k_{y}$ are wave numbers along the $x$-and $y$-directions respectively, $k\left(=\sqrt{k_{x}^{2}+k_{y}^{2}}\right)$ is the resultant wave number and $n$ is the growth rate, which is, in general, a complex constant.

Using expression (2.14), Eqs (2.11) - (2.13) in a non-dimensional form become

$$
\begin{aligned}
& \sigma\left(D^{2}-a^{2}\right) W+\frac{g \alpha d^{2}}{v} a^{2} \Theta+\left(T_{A}^{1 / 2} d\right) D Z=\left[1-F\left(D^{2}-a^{2}\right)\right]\left(D^{2}-a^{2}\right)^{2} W, \\
& {\left[\left\{1-F\left(D^{2}-a^{2}\right)\right\}\left(D^{2}-a^{2}\right)-\sigma\right] Z=-\frac{T_{A}^{1 / 2}}{d} D W,} \\
& \left(D^{2}-a^{2}-p_{1} \sigma\right) \Theta=-\left(\frac{G-1}{G}\right) \frac{\beta d^{2}}{\kappa} W
\end{aligned}
$$


where we have put $a=k d, \sigma=\frac{n d^{2}}{v}, x / d=x^{*}, y / d=y^{*}, z / d=z^{*}$ and $D=\frac{d}{d z^{*}}$. Here $p_{1}=\frac{v}{\kappa}$ is the Prandtl number, $F=\frac{\mu^{*}}{\rho_{0} d^{2} v}$ is the dimensionless couple-stress parameter, $G=\frac{c_{p} \beta}{g}$ is the dimensionless compressibility parameter and $\mathrm{T}_{\mathrm{A}}=\frac{4 \Omega^{2} d^{4}}{v^{2}}$ is the Taylor number.

Eliminating $\Theta, Z$ between Eqs (2.15)-(2.17), we obtain

$$
\begin{aligned}
& \sigma\left(D^{2}-a^{2}\right)\left(D^{2}-a^{2}-p_{1} \sigma\right)\left[\left\{1-F\left(D^{2}-a^{2}\right)\right\}\left(D^{2}-a^{2}\right)-\sigma\right] W-R a^{2}\left(\frac{G-1}{G}\right), \\
& {\left[\left\{1-F\left(D^{2}-a^{2}\right)\right\}\left(D^{2}-a^{2}\right)-\sigma\right] W-T_{A}\left(D^{2}-a^{2}-p_{1} \sigma\right) D^{2} W=} \\
& =\left(D^{2}-a^{2}-p_{1} \sigma\right)\left[\left\{1-F\left(D^{2}-a^{2}\right)\right\}\left(D^{2}-a^{2}\right)-\sigma\right]\left\{1-F\left(D^{2}-a^{2}\right)\right\}\left(D^{2}-a^{2}\right)^{2} W .
\end{aligned}
$$

We now assume that the fluid layer is confined between two free boundaries. The case is of artificial nature, but due to mathematical simplicity it enables us to show the effect of rotation on the couple-stress fluid analytically. The appropriate boundary conditions, with respect to which Eqs (2.15)-(2.17) must be solved, are

$$
W=D^{2} W=0, \quad \Theta=0, \quad D Z=0 \quad \text { at } \quad z=0 \quad \text { and } \quad z=1 .
$$

Equations (2.8), (2.14) and (2.19) imply that

$$
D^{4} W=0 \quad \text { at } \quad z=0 \quad \text { and } \quad 1 .
$$

Dropping the caps for convenience and using the above boundary conditions, it can be shown that all the even order derivatives of $W$ must vanish on the boundaries and hence the proper solution of Eq.(2.18) characterizing the lowest mode is

$$
W=W_{0} \sin \pi z
$$

where $W_{0}$ is a constant

Substituting the proper solution (2.21) in Eq.(2.18), we obtain the dispersion relation

$$
\begin{gathered}
(1+x)^{2}\left(1+x+i p_{l} \sigma_{1}\right)\left\{1+F_{l}(1+x)\right\}\left[(1+x)\left\{1+F_{l}(1+x)\right\}+i \sigma_{1}\right]+ \\
R_{l}=\left(\frac{G}{G-1}\right) \frac{+i \sigma_{l}(1+x)\left(1+x+i p_{1} \sigma_{1}\right)\left[(1+x)\left\{1+F_{l}(1+x)\right\}+i \sigma_{1}\right]+T_{1}\left(1+x+i p_{1} \sigma_{1}\right)}{x\left[(1+x)\left\{1+F_{l}(1+x)\right\}+i \sigma_{1}\right]}
\end{gathered}
$$

where

$$
\frac{\sigma}{\pi^{2}}=i \sigma_{1}, \quad R_{1}=\frac{R}{\pi^{4}}, \quad T_{1}=\frac{T_{A}}{\pi^{4}}, \quad x=\frac{a^{2}}{\pi^{2}} \quad \text { and } \quad F_{l}=\pi^{2} F
$$




\section{Stationary convection}

When the instability sets in as stationary convection, the marginal state will be characterized by $\sigma=$ 0 Eq.(2.22) reduces to

$$
R_{l}=\left(\frac{G}{G-1}\right)\left[\frac{(1+x)^{3}\left\{1+F_{1}(1+x)\right\}^{2}+T_{1}}{x\left\{1+F_{1}(1+x)\right\}}\right]
$$

Equation (3.1) expresses the modified Rayleigh number $\mathrm{R}_{1}$ as a function of the dimensionless wave number $x$ and the parameters $G, F_{1}$ and $T_{1}$. For fixed $F_{1}$ and $T_{1}$, let $G$ (accounting for the compressibility effects) also be kept fixed. Then we find that

$$
\overline{R_{c}}=\left(\frac{G}{G-1}\right) R_{c}
$$

where $\overline{R_{c}}$ and $\mathrm{R}_{\mathrm{c}}$ denote, respectively, the critical Rayleigh numbers in the presence and absence of compressibility. $G>1$ is relevant here.

The effect of compressibility is thus to postpone the onset of thermal instability.

Equation (3.1) yields

$$
\frac{d R_{1}}{d T_{1}}=\left(\frac{G}{G-1}\right) \frac{1}{x\left\{1+F_{1}(1+x)\right\}},
$$

which implies that rotation has postponed the onset of convection. Equation (3.1) also yields

$$
\frac{d R_{1}}{d F_{1}}=\left(\frac{G}{G-1}\right)\left[\frac{(1+x)^{4}}{x}-\frac{T_{1}(1+x)}{x\left\{1+F_{l}(1+x)\right\}^{2}}\right] .
$$

In the absence of rotation $\left(T_{I}=0\right), d R_{l} / d F_{1}$ is positive which means that the couple-stress has postponed the onset of convection. For a rotating system, the couple-stress still postpones the onset of convection if $T_{1}<(1+x)^{3}\left\{1+F_{1}(1+x)\right\}^{2}$ as well as hastens the onset of convection if $T_{1}>(1+x)^{3}\left\{1+F_{1}\right.$ $(1+x)\}^{2}$.

\section{Stability of the system and oscillatory modes}

Here we examine the possibility of oscillatory modes, if any, as a stability problem due to the presence of rotation. Multiplying Eq.(2.15) by $W^{*}$, the complex conjugate of $W$, integrating over the range of $\mathrm{z}$ and making use of Eqs (2.16) and (2.17) together with the boundary conditions (2.19), and (2.20), we obtain

$$
\sigma I_{1}-\frac{g \alpha \kappa a^{2}}{v \beta}\left(\frac{G}{G-1}\right)\left(I_{2}+p_{1} \sigma^{*} I_{3}\right)+d^{2}\left(I_{4}+F I_{5}\right)+d^{2} \sigma^{*} I_{6}-I_{7}-F I_{8}=0
$$

where 


$$
\left.\begin{array}{c}
I_{1}=\int_{0}^{1}\left(|D W|^{2}+a^{2}|W|^{2}\right) d z, \\
I_{2}=\int_{0}^{1}\left(|D \Theta|^{2}+a^{2}|\Theta|^{2}\right) d z, \\
I_{3}=\int_{0}^{1}|\Theta|^{2} d z \\
I_{4}=\int_{0}^{1}\left(|D Z|^{2}+a^{2}|Z|\right) d z \\
I_{5}=\int_{0}^{1}\left(\left|D^{2} Z\right|^{2}+2 a^{2}|D Z|^{2}+a^{4}|Z|^{2}\right) d z \\
I_{7}=\int_{0}^{1}\left(\left|D^{2} W\right|^{2}+2 a^{2}|D W|^{2}+a^{4}|W|^{2}\right) d z \\
I_{8}=\int_{0}^{1}(|Z| d z, \\
\left.\left.I_{0}^{3} W\right|^{2}+3 a^{2}\left|D^{2} W\right|^{2}+3 a^{4}|D W|^{2}+a^{6}|W|^{2}\right) d z
\end{array}\right\}
$$

which are all positive definite. Putting $\sigma=\sigma_{r}+i \sigma_{i}$ in Eq.(4.1) and equating real and imaginary parts, we obtain

$$
\sigma_{r}\left[I_{1}-\frac{g \alpha \kappa a^{2}}{v \beta}\left(\frac{G}{G-1}\right) p_{1} I_{3}+d^{2} I_{6}\right]=-\left[\frac{g \alpha \kappa a^{2}}{v \beta}\left(\frac{G}{G-1}\right) I_{2}+d^{2}\left(I_{4}+F I_{5}\right)+I_{7}+I_{8}\right],
$$

and

$$
\sigma_{i}\left[I_{1}+\frac{g \alpha \kappa a^{2}}{v \beta}\left(\frac{G}{G-1}\right) p_{1} I_{3}-d^{2} I_{6}\right]=0
$$

Equation (4.4) yields that $\sigma_{i}=0$ or $\sigma_{i} \neq 0$, which means that the modes may be non-oscillatory or oscillatory. In the absence of rotation, Eq.(4.4) reduces to

$$
\sigma_{i}\left[I_{1}+\frac{g \alpha \kappa a^{2}}{v \beta}\left(\frac{G}{G-1}\right) p_{1} I_{3}\right]=0
$$

and the terms in bracket are positive definite. Thus $\sigma_{i}=0$, which means that oscillatory modes are not allowed and the principle of exchange of stabilities is satisfied in the absence of rotation. This result is true for compressible, couple-stress fluids as well as for incompressible Newtonian fluids [Chandrasekhar [4]], in the absence of rotation. The presence of rotation brings oscillatory modes (as $\sigma_{i}$ may not be zero) which were non-existent in its absence. Equation (4.3) simply shows that there may be stability or instability in the presence of rotation in compressible, couple-stress fluids which is also true in the absence of rotation as well as in incompressible, Newtonian fluids [Chandrasekhar [4]]. 


\section{The overstable case}

Here we discuss the possibility of whether instability may occur as overstability. Since for overstability we wish to determine the critical Rayleigh number for the onset of instability via a state of pure oscillations, it suffices to find conditions for which (2.22) will admit solutions with $\sigma_{1}$ real. Equating the real and imaginary parts of Eq.(2.22) and eliminating $R_{l}$ between them, we obtain

$$
\begin{aligned}
& \sigma_{1}^{2}\left(1+p_{1}+p_{1} F_{1} \alpha\right) \alpha+\left[\alpha^{6} p_{1} F_{1}^{3}+\alpha^{5} F_{1}^{2}\left(3 p_{1}+1\right)+\alpha^{4} F_{1}\left(2+3 p_{1}\right)+\right. \\
& \left.+\alpha^{3}\left(1+p_{1}\right)+\alpha p_{1} T_{1} F_{1}+T_{1}\left(p_{1}-1\right)\right]=0
\end{aligned}
$$

where we have put $1+x=\alpha$.

As $\sigma_{l}$ is real for overstability, $\sigma_{l}^{2}$ is positive. It is evident from Eq.(5.1) that this is clearly impossible if

$$
\begin{array}{ll} 
& p_{1}>1, \\
\text { i.e., } & \text { if } \kappa<v .
\end{array}
$$

$\kappa<v$ is, therefore, a sufficient condition for the non-existence of overstability, the violation of which does not necessarily imply the occurrence of overstability. The sufficient condition $\kappa<v$ for the non-existence of overstability is found to be the same for compressible, couple-stress fluids as well as for incompressible, Newtonian fluids (Chandrasekhar, [4]), in presence of rotation and heated from below.

\section{Effect of magnetic field}

\subsection{Formulation of the problem and perturbation equations}

Here we consider an infinite, horizontal, compressible, electrically conducting couple-stress fluid layer of thickness $\mathrm{d}$, heated from below. This layer is acted on by a uniform vertical magnetic field $\boldsymbol{H}(0,0, H)$ and gravity field $\boldsymbol{g}(0,0,-g)$. Let $\boldsymbol{q}(u, v, w), \theta, \delta p$ and $\delta \rho$ denote, respectively, the perturbations in velocity $(0,0,0)$, temperature $T$, pressure $p$, and density $\rho$. Then the linearized perturbation equations relevant to the problem [Stokes [7], Sharma [13]] are

$$
\begin{aligned}
& \frac{\partial \boldsymbol{q}}{\partial t}=-\frac{1}{\rho_{m}} \nabla \delta p-g \alpha \theta+\left(v-\frac{\mu}{\rho_{m}} \nabla^{2}\right) \nabla^{2} \boldsymbol{q}+\frac{\mu_{e}}{4 \pi \rho_{m}}(\nabla \times \boldsymbol{h}) \times \boldsymbol{H}, \\
& \nabla \cdot \boldsymbol{q}=0, \\
& \nabla . \boldsymbol{h}=0, \\
& \frac{\partial \boldsymbol{h}}{\partial t}=(\overrightarrow{H . \nabla}) \boldsymbol{q}+\eta \nabla^{2} \boldsymbol{h},
\end{aligned}
$$

together with Eq.(2.7). Equations (2.7), (6.1)-(6.4) give 


$$
\begin{aligned}
& \frac{\partial}{\partial t} \cdot\left(\nabla^{2} w\right)-\left(v-\frac{\mu}{\rho_{m}} \nabla^{2}\right) \nabla^{4} w-g \alpha\left(\frac{\partial^{2}}{\partial x^{2}}+\frac{\partial^{2}}{\partial y^{2}}\right) \theta-\frac{\mu_{e}}{4 \pi \rho_{m}} \frac{\partial}{\partial z} \nabla^{2} h_{z}=0, \\
& \left(\frac{\partial}{\partial t}-\eta \nabla^{2}\right) h_{z}=H \frac{\partial w}{\partial z}
\end{aligned}
$$

together with Eq.(2.13).

\section{The dispersion relation}

Using expression (2.14), Eqs (6.5)-(6.6) and (2.13), in a non-dimensional form, we have

$$
\begin{aligned}
& \sigma\left(D^{2}-a^{2}\right) W+\frac{g \alpha d^{2} a^{2}}{v} \Theta=\left[1-F\left(D^{2}-a^{2}\right)\right]\left[\left(D^{2}-a^{2}\right)^{2} W\right]+ \\
& +\frac{\mu_{e} H d}{4 \pi \rho_{m} v}\left(D^{2}-a^{2}\right) D K \\
& \left(D^{2}-a^{2}-p_{2} \sigma\right) K=-\frac{H d}{\eta} D W,
\end{aligned}
$$

together with Eq.(2.16).

Using the boundary conditions Eqs (2.19) - (2.21), eliminating $\Theta, K$ between Eqs $(7.1)-(7.2)$ and (2.16), we get

$$
\begin{aligned}
& \sigma\left\{1-F\left(D^{2}-a^{2}\right)\right\}\left[D^{2}-a^{2}\right]^{2}\left[D^{2}-a^{2}-p_{1} \sigma\right]\left[D^{2}-a^{2}-p_{2} \sigma\right] W+ \\
& +R a^{2} \frac{G-1}{G}\left(D^{2}-a^{2}-p_{2} \sigma\right) W-Q\left(D^{2}-a^{2}\right)\left(D^{2}-a^{2}-p_{1} \sigma\right) D^{2} W=0
\end{aligned}
$$

where $\mathrm{R}=\frac{g \alpha \beta d^{4}}{\nu \kappa}$ is the Rayleigh number and $\mathrm{Q}=\frac{\mu_{e} H^{2} d^{2}}{4 \pi \rho_{m} \nu \eta}$ is the Chandrasekhar number.

Using Eq.(2.21), Eq.(7.3) yields

$$
R_{l}=\frac{G}{G-1} \frac{(1+x)\left(1+x+i p_{1} \sigma_{1}\right)\left[\left(1+x+i p_{2} \sigma_{l}\left\{i \sigma_{1}+\left(1+F_{1} \overline{1+x)}(1+x)\right\}+Q_{1}\right]\right.\right.}{x\left(1+x+i p_{2} \sigma_{1}\right)}
$$

where $\quad R_{l}=\frac{R}{\pi^{4}}, \quad Q_{l}=\frac{Q}{\pi^{2}}, \quad a^{2}=\pi^{2} x, \quad i \sigma_{l}=\frac{\sigma}{\pi^{2}} \quad$ and $\quad F_{l}=\pi^{2} F$.

\section{Stationary convection}

When the instability sets in as stationary convection, the marginal state will be characterized by $\sigma=0$ and Eq.(7.4) reduces to 


$$
R_{l}=\left(\frac{G}{G-1}\right) \frac{1+x}{x}\left[(1+x)^{2}+F_{1}(1+x)^{3}+Q_{1}\right]
$$

Equation (8.1) yields

$$
\begin{aligned}
& \frac{d R_{1}}{d F_{1}}=\frac{G}{G-1} \frac{(1+x)^{4}}{x}, \\
& \frac{d R_{1}}{d Q_{1}}=\frac{G}{G-1} \frac{1+x}{x},
\end{aligned}
$$

which are always positive. The couple-stress and magnetic field, thus, postpones the onset of convection.

For fixed $Q_{1}$ and $F_{1}$, let $G$ (accounting for the compressibility effects) be also kept fixed in Eq.(8.1). Then we find that the result is similar as in Eq.(3.2).

The results have been also shown graphically . In Fig.1, the variation of the Rayleigh number $R_{I}$ with the wave number $x$, for $Q_{1}=100$ and $G=10$ is considered when the couple-stress parameter $F_{l}$ is varied. It is clear from the graph that the Rayleigh number $\mathrm{R}_{1}$ increases with the increase in the value of $F_{1}$ thus implying a stabilizing effect of the couple-stress parameter. Figure 2 shows that the variation of Rayleigh number $\mathrm{R}_{1}$ with the wave number $x$ for $F_{l}=2$ and $G=10$, indicating that $Q_{1}$ is varied. It is clear from the graph that with the increase in the value of $Q_{l}$, there is an increase in the value of the Rayleigh number $\mathrm{R}_{1}$ which suggests that the presence of magnetic field causes the stabilizing effect for $G>1$.

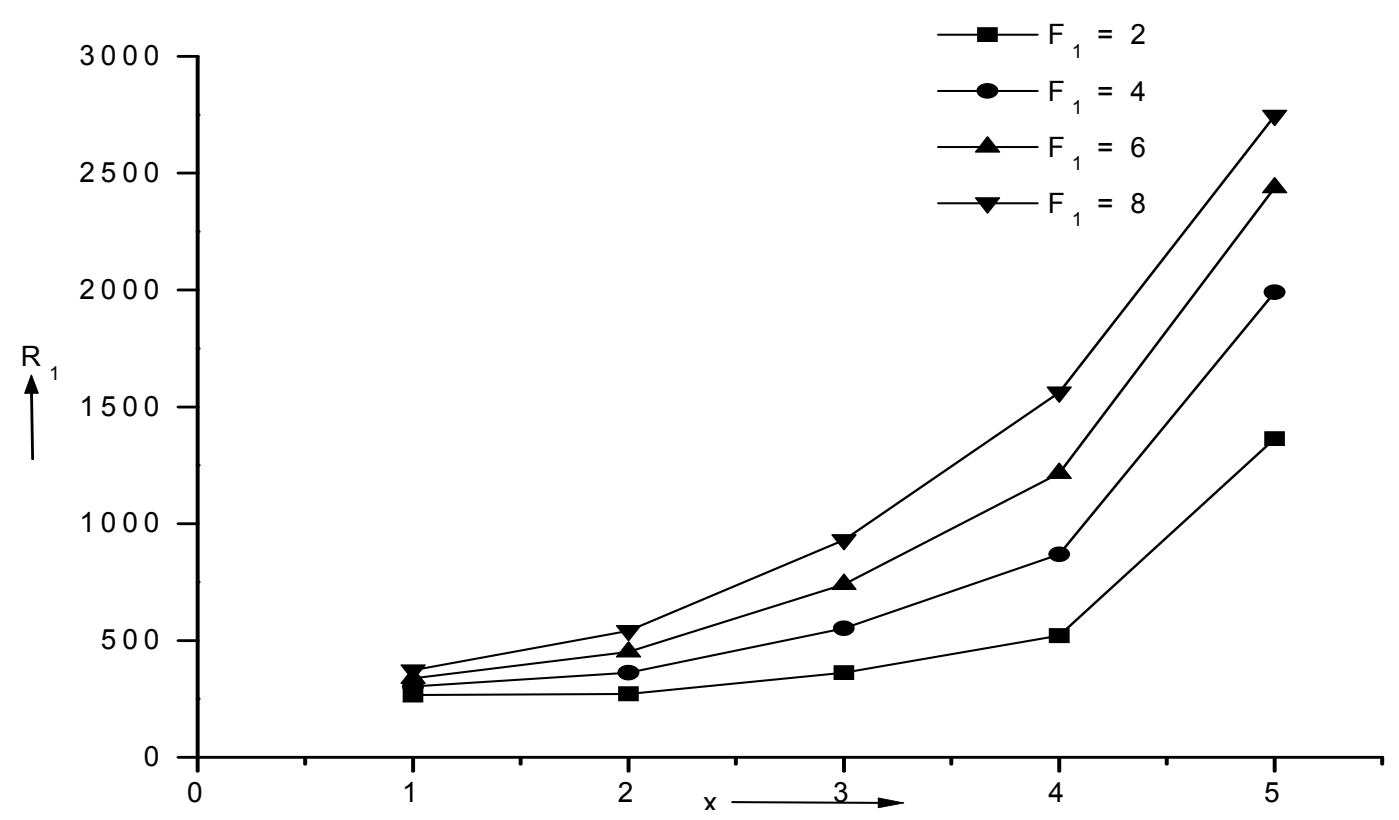

Fig.1. Variation of the Rayleigh number $\mathrm{R}_{1}$ with the wave number $x$ for $Q_{1}=100$ and $G=10$. 


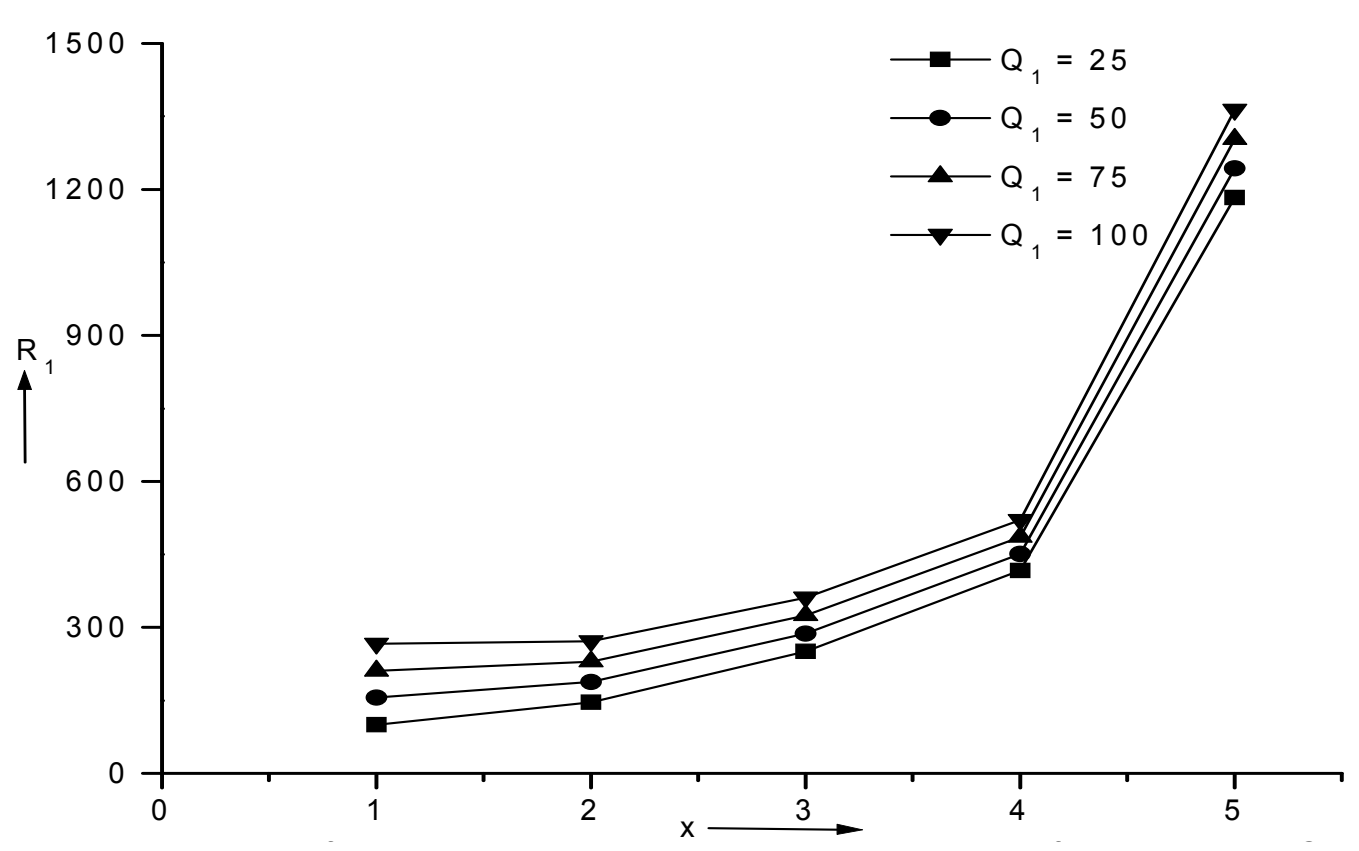

Fig.2. Variation of the Rayleigh number $\mathrm{R}_{1}$ with the wave number $x$ for $F_{1}=2$ and $G=10$.

\section{Some important theorems}

Theorem 1: The system is stable for $G<1$.

Proof: Multiplying Eq.(6.5) by $W^{*}$, the complex conjugate of $W$ and using Eqs (6.6), (2.13) and boundary conditions (2.19) - (2.21), we obtain

$$
\sigma I_{1}-\frac{g \alpha a^{2} \kappa}{v \beta}\left(\frac{G}{G-1}\right)\left(I_{2}+p_{1} \sigma^{*} I_{3}\right)+\left(I_{6}+F I_{7}\right)+\frac{\mu_{e} \eta}{4 \pi \rho_{m} \nu}\left[I_{4}+\sigma^{*} p_{2} I_{5}\right]=0
$$

where $I_{1}-I_{7}$ are similar as in Eq.(4.2), and $\sigma^{*}$ is the complex conjugate of $\sigma$. The integrals $I_{1}-I_{7}$ are all positive definite. Putting $\sigma=\sigma_{r}+i \sigma_{i}$ in Eq.(9.1) and equating real and imaginary parts, we obtain

$$
\sigma_{r}\left[I_{1}-\frac{g \alpha a^{2} \kappa}{v \beta} p_{1} I_{3} \frac{G}{G-1}+\frac{\eta \mu_{e}}{4 \pi \rho_{m} \nu} p_{2} I_{5}\right]=-\left[-\frac{g \alpha a^{2}}{v \beta}\left(\frac{G}{G-1}\right) \kappa I_{2}+\frac{\mu_{e} \eta}{4 \pi \rho_{m} v} I_{4}+I_{6}+F I_{7}\right]
$$

and

$$
\sigma_{i}\left[I_{1}+\frac{g \alpha a^{2} \kappa}{v \beta}\left(\frac{G}{G-1}\right) p_{1} I_{3}-\frac{\eta \mu_{e}}{4 \pi \rho_{m} v} p_{2} I_{5}\right]=0 .
$$

It is evident from Eq.(9.2) that if $G<1, \sigma_{r}$ is negative meaning thereby the stability of the system.

Theorem 2: The modes may be oscillatory or non-oscillatory in contrast to the case of no magnetic field where modes are non-oscillatory, for $G>1$. 
Proof: Equation (9.3) yields that $\sigma_{i}=0$ or $\sigma_{i} \neq 0$ which means that modes may be non-oscillatory or oscillatory. In the absence of the magnetic field, Eq.(9.3) gives

$$
\sigma_{i}\left[I_{1}+\frac{g \alpha a^{2} \kappa}{v \beta} \frac{G}{G-1} p_{1} I_{3}\right]=0,
$$

and the terms in brackets are positive definite when $G>1$. Thus $\sigma_{i}=0$, which means that oscillatory modes are not allowed and the principle of exchange of stabilities is satisfied in the absence of the magnetic field. This result is true for compressible, couple-stress fluids as well as for incompressible Newtonian fluids [Chandrasekhar [4]] in the absence of the magnetic field. The presence of the magnetic field brings oscillatory modes (as $\sigma_{i}$ may not be zero) which were non-existent in its absence. Equation (9.2) simply shows that there may be stability or instability in the presence of the magnetic field in compressible couplestress fluids, which is also true in the absence of the magnetic field, as well as in incompressible, Newtonian fluids [Chandrasekhar [4]].

Theorem 3: $\kappa<\eta$ is the sufficient condition for the non-existence of overstability.

Proof: For overstability, we put $\frac{\sigma}{\pi^{2}}=i \sigma_{l}$ where $\sigma_{l}$ is real and Eq.(7.4) can be written as

$$
\begin{aligned}
& R_{1} x\left(1+x+i p_{2} \sigma_{1}\right)= \\
& =\frac{G}{G-1}(1+x)\left(1+x+i p_{1} \sigma_{1}\right)\left[\left(1+x+i p_{2} \sigma_{1}\right)\left\{i \sigma_{1}+\left(1+F_{1} \overline{1+x}\right)(1+x)\right\}+Q_{1}\right] .
\end{aligned}
$$

Since for overstability we wish to determine the critical Rayleigh number for the onset of instability via a state of pure oscillations, it suffices to find conditions for which (9.5) will admit solutions with $\sigma_{l}$ real. Equating real and imaginary parts of Eq.(9.5) and eliminating $R_{l}$ between them, we obtain the equation

$$
\begin{aligned}
& p_{1} p_{2}^{2} \alpha \sigma_{1}^{2}+\left[p_{2} \alpha^{3}\left(p_{1}-p_{2}\right)+p_{2} F_{1} \alpha\left(p_{1}-p_{2}\right)\right] \sigma_{1}+ \\
& +\left[\alpha^{4}\left(p_{1}-p_{2}\right)+\alpha^{5} F_{1}\left(p_{1}-p_{2}\right)+Q_{1} \alpha\left(p_{1}-p_{2}\right)\right]=0,
\end{aligned}
$$

which may be presented in the following form

$$
A \sigma_{1}^{2}+B \sigma_{1}+C=0
$$

where

$$
\begin{aligned}
& A=p_{1} p_{2}^{2} \alpha \\
& B=\left(-\alpha p_{2}-p_{2}^{2} \alpha^{3}-p_{2}^{2} \alpha^{4} F_{1}+p_{2} \alpha^{3}+p_{1} p_{2} \alpha^{3}+p_{1} p_{2} F_{1} \alpha\right)= \\
& =p_{2} \alpha^{3}\left(p_{1}-p_{2}\right)+p_{2} F_{1} \alpha\left(p_{1}-p_{2}\right) \\
& C=\left(-\alpha^{4} p_{2}-F_{1} \alpha^{5} p_{2}-Q \alpha^{2} p_{2}+p_{1} \alpha^{3}+p_{1} \alpha^{4}+p_{1} \alpha^{5} F_{1}+p_{1} Q_{1} \alpha\right)= \\
& =\alpha^{4}\left(p_{1}-p_{2}\right)+\alpha^{5} F_{1}\left(p_{1}-p_{2}\right)+Q_{1} \alpha\left(p_{1}-p_{2}\right) .
\end{aligned}
$$


and

$$
1+x=\alpha
$$

Equation (9.6) is quadratic in $\sigma_{l}$, as $\sigma_{l}$ is real for overstability, $\sigma^{2}{ }_{l}$ is positive. It is evident from Eq.(9.6) that $p_{1}>p_{2}$, thus implying

$$
\frac{v}{\kappa}>\frac{v}{\eta}
$$

i.e., $\kappa<\eta$ is, therefore, a sufficient condition for the non-existence of overstability, the violation of which does not necessarily imply the occurrence of overstability. The sufficient condition for the non-existence of overstability is found to be the same for compressible, couple-stress fluids as well as for incompressible Newtonian fluids [Chandrasekhar, [4]], in the presence of the magnetic field, which is heated from below.

\section{Conclusion}

The effect of a uniform vertical rotation and uniform magnetic filed on thermal convection in a layer of a couple-stress fluid heated from below is considered (separately) in the present paper. The synovial fluid is the natural lubricant of joints of the vertebrates. The detailed description of the joint lubrication has very important practical implications. Practically, all diseases of joints are caused by or connected with a malfunction of lubrication. The main conclusions from the analysis (i.e., the effect of uniform vertical rotation and the effect of uniform magnetic field), are as follows:

\section{Effect of Rotation}

(1) For the case of stationary convection, uniform vertical rotation postpones the onset of convection whereas the couple-stress parameter postpones as well as hastens the onset of convection, while in the absence of rotation the couple-stress parameter always postpones the onset of convection.

(2) It is observed that vertical uniform rotation introduces oscillatory modes in the system, which were nonexistent in its absence. In the absence of rotation oscillatory modes are not allowed and the principle of exchange of stabilities is valid.

(3) The condition

$p_{1}>1$ i.e. $\kappa<\mathrm{U}$ is, therefore the sufficient condition for the non-existence of overstability, the violation of which does not necessarily involve an occurrence of overstability.

\section{Effect of Magnetic Field}

(1) For the case of stationary convection, the couple-stress parameter and uniform magnetic field postpone the onset of convection.

(2) It is observed from Figs 1-2 that the couple-stress parameter and uniform magnetic field have a stabilizing effect on the system.

(3) It is also observed that the uniform magnetic field introduces oscillatory modes in the system, which were non-existent in its absence and in the absence of the magnetic field, oscillatory modes are not allowed and the principle of exchange of stabilities is valid.

(4) The condition $\frac{\mathrm{U}}{\kappa}>\frac{\mathrm{v}}{\eta}$ i.e $\kappa<\eta$ is, therefore, a sufficient condition for the non-existence of overstability.

(5) The effect of compressibility postpones the onset of convection, when $G>1$ in both cases (i.e., uniform vertical rotation as well as uniform magnetic field). 


\section{Nomenclature}

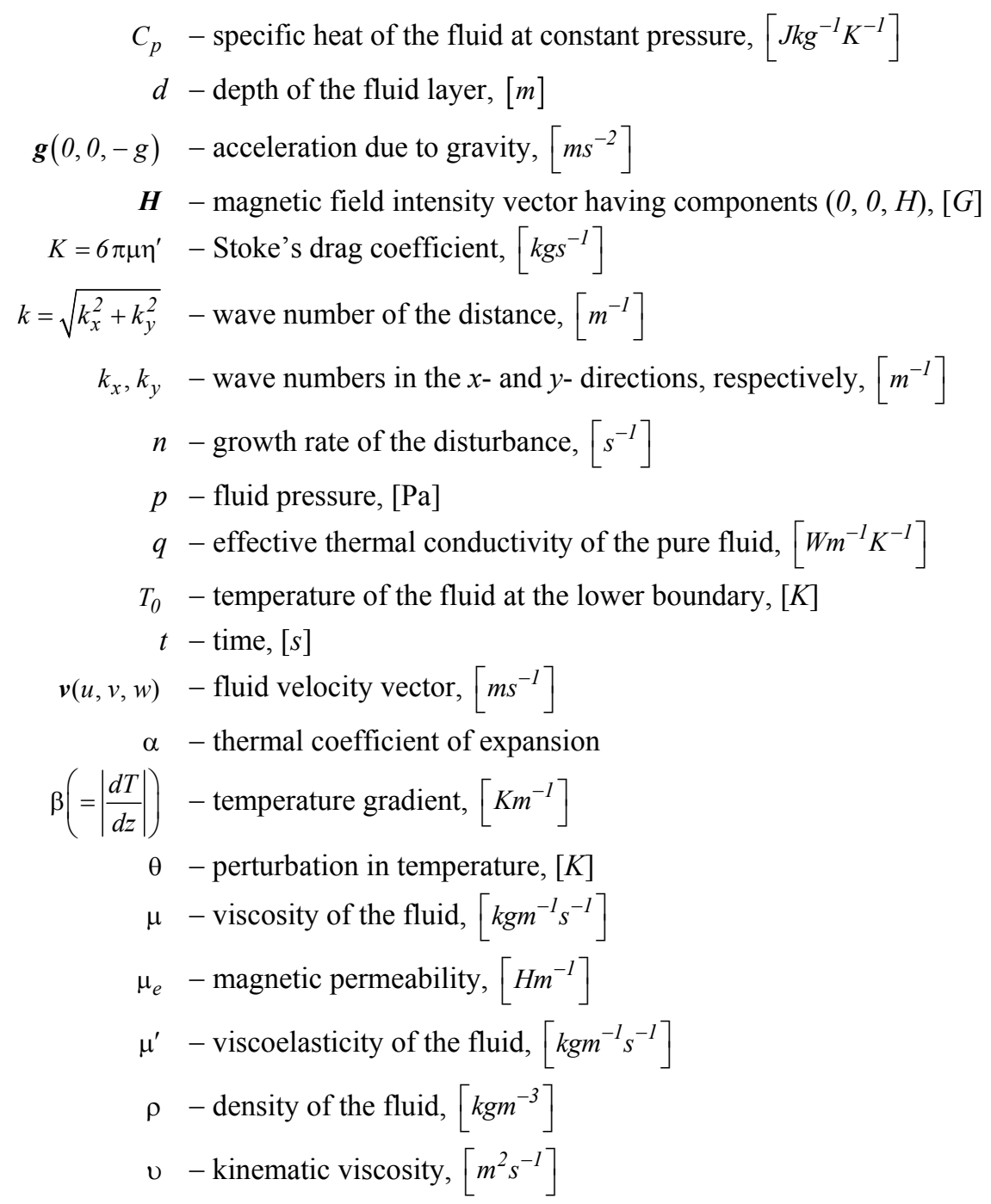

\section{References}

[1] Bénard H. (1900): Les Tourbillons cellularies dans une nappe liquide. - Revue Gene'rale des Sciences Pures et Applique'es, vol.11, pp.1261-1271, 1309-1328.

[2] Rayleigh L. (1916): On convection currents in a horizontal layer of fluid, when the higher temperature is on the underside. - Phil. Mag., vol.32, pp.529-546.

[3] Jeffreys H. (1926): The stability of a layer of fluid heated from below. - Phil. Mag., vol.2, pp.833-844.

[4] Chandrasekhar S. (1981): Hydrodynamic and Hydromagnetic Stability. - Oxford: Clarendon Press.

[5] Lai W.M., Kuei S.C. and Mow V.C. (1978): Rheological equations for synovial fluids. - J. Biomechanical Engng., vol.100, pp.169-186.

[6] Walicka A. (1994): Micropolar flow in a slot between rotating surfaces of revolution. - Zielona Gora: TU Press.

[7] Stokes V.K. (1966): Couple-stresses in fluids. - Phys. Fluids, vol.9, pp.1709-1715. 
[8] Nason P., Schumaker V., Halsalt B. and Schwedes J. (1969): Formation of a streaming convective disturbance which may occur at one gravity during preparation of samples for zone centrifugation. - Biopolymers, vol.7, pp.241-249.

[9] Spiegel E.A and Veronis G. (1960): On the Boussinesq approximation for a compressible fluid. - J. Astrophys., vol.131, pp.442-444.

[10] Walicki E. and Walicka A. (1999): Inertia effect in the squeeze film of a couple-stress fluid in biological bearings. - Appl. Mech. Engng., vol.2, pp.363-373.

[11] Goel A.K., Agarwal S.C. and Agarwal G.S. (1999): Hydromagnetic stability of an unbounded couple stress binary fluid mixture having vertical temperature and concentration gradients with rotation. - Indian J. Pure Appl. Math., vol.30, No10, pp.991-1001.

[12] Kumar P., Lal R. and Sharma P. (2004): Effect of rotation on thermal instability in couple-stress elastico-viscous fluid. - Z. Naturforsch., vol.59a, pp.407-411.

[13] Sharma R.C. (1977): Thermal instability in compressible fluids in the presence of rotation and magnetic field. - J. Math. Anal. Appl., vol.60, pp.227-235.

[14] Singh M. and Kumar P. (2011): Magneto and incompressible rotatory thermosolutal convection in couple-stress fluid in porous medium. - Journal of Porous Medium, vol.14, No.7, pp.637-648.

[15] Singh M., Mehta C.B. and Gangta S. (2012): Thermosolutal convection compressible rotating couple-stress fluid in presence of magnetic field. - Advances in Applied Science Research (USA), vol.3, No.6, pp.3459-3468.

[16] Singh M. and Kumar P. (2012): Thermosolutal convection in a couple-stress fluid in uniform vertical magnetic field. - Z. Naturforsch, vol.67a, pp.275-281.

Received: December 28, 2016

Revised: October 16, 2017 